\title{
Fetal Lung Maturation IV: The Release of Phosphatidic Acid Phosphohydrolase and Phospholipids into the Human Amniotic Fluid
}

\author{
JUAN M. JIMENEZ AND JOHN M. JOHNSTON
}

From the Cecil H. and Ida Green Center for Reproductive Biology Sciences, Departments of Obstetrics and Gynecology and Biochemistry, The University of Texas Health Science Center at Dallas, Southwestern Medical School, Dallas, Texas, U. S. A.: and The University of Texas Health Science Center at Dallas, Southwestern Medical School, Department of Obstetrics and Gynecology, 5323 Harry Hines Blvd., Dallas, Texas 75235 (U.S.A.)

\section{Extract}

The relationship between surface active material and phosphatidic acid phosphohydrolase (PAPase) activity was examined in human amniotic fluid after centrifugation at $105,000 \times \mathrm{g}$ for one hour. The distibution of PAPase activity between the pellet and supernatant fractions was similar to that of phospholipids in the amniotic fluid. The majority of the PAPase activity was found to be associated with the pellet fraction. Similarly, $82 \%$ of the phospholipid was also present in this fraction. Of the total palmitate present in the original sample, approximately $90 \%$ was found in the pellet after centrifugation. Palmitic acid constituted $75 \%$ of all fatty acids present in the phosphatidylcholine identified in the pellet. By sharp contrast only $4 \%$ of the total protein was pelletized by centrifugation. The pelletized fraction isolated from amniotic fluid after $105,000 \times \mathrm{g}$ centrifugation closely resembles the lamellar bodies in: $(1)$ its phospholipid pattern; (2) palmitate concentration in phosphatidylcholine; and, (3) the presence of PAPase activity with a high specific activity. Collectively, these findings lend strong support to the concept that a lamellarlike body is released from fetal lung into the amniotic fluid.

\section{Speculation}

Inadequate production of surface active material by the lung plays an important role in the pathogenesis of respiratory distress syndrome of the newborn. The measurement of key enzymes responsible for the regulation of phosphatidylcholine synthesis would be useful as another index of fetal lung maturity. The response to prenatal therapy to induce surfactant synthesis or release could be monitored by the assay of these enzymes in the amniotic fluid and help to define the normal intrauterine stimuli for the surge of phosphatidylcholine synthesis. 
Several investigators have found that the maturation process of the fetal lung is associated with an increased production of a lipoprotein possessing surface-active properties. The major phospholipid of this lipoprotein is dipalmitoyl-phosphatidylcholine (DPPC); moreover this phospholipid is thought to be responsible for most of the surface-active properties of the complex. The several enzymes that catalyze the blosynthesis of DPPC have been studied in both adult and developing fetal lung (3). We have found that phosphatidic acid phosphohydrolase activity [phosphatidate phosphohydrolase (EC. 3.1.3.4)] (PAPase), a key enzym begins to increase in fetal rabbit lung on day 26 of gestation and its activity continues to increase and reaches adult levels just prior to parturition (18). In contrast to the observed increase in PAPase activity, the activity of the enzyme lung of fetal rabbits did not change during the course of gestation $(5,18)$. Tissue specificity for the increase in PAPase activity in the lung was suggested by the finding in liver PAPase activity did not increase during fetal development (18). We have also demonstrated PAPase activity in human amniotic fluid (9). (18). We have also demonstrated PAPase activity in human amnlotic fluid (9). fluid obtained from pregnancies of more than 35 weeks than in amniotic fluid fluid obtained from pregnancies of more than 35 weeks than in amniotic fluid
from pregnancles before the 32 nd week of gestation. In a study of the sequen from pregnancies before the 32 nd week of gestation. In a study of the sequen amniocentesis were performed beginning at 30 weeks of gestation a continuing Increase in activity was observed that reached peak activity by the 37 th week. The observed increase in amniotic fluid PAPase activity preceded the "surge" of acetone precipitable phosphatldyicholine by approximately one and one half weeks. Also, the speciflc activity of PAPase in the nasopharingeal fluid was found to be 3 to 4 times greater than that of the amniotic fluid (10). These found to be 3 to 4 times greater than that of the amniotic fluid $(10)$. These findings support the concept that PAPase originates in the fetal lung. the surface-active material and PAPase activity in human amniotic fluid.

\section{METHODS AND MATERIALS}

\section{Amniotic Fluld Fractionation}

Twenty-five $\mathrm{ml}$ of amniotic fluid were collected at the time of cesarean section from gravidas at term. To remove intact cells and cell debris, the samples were chilled immediately and centrifuged at $4^{\circ} \mathrm{C}$ at $700 \times \mathrm{g}$ for 5 is referred to subsequently as the original amnlotic fluid sample. An aliquot of the original sample was centrifuged at $105,000 \times \mathrm{g}$ for 60 minutes at $4^{\circ} \mathrm{C}$. The supernatant fraction and the pellet were analyzed separately.

\section{Gas Liquid Chromatography}

The IIpids were extracted from amniotic fluid by the method described by Folch et al.. (4). The chloroform was removed by evaporation under nitrogen at $25^{\circ} \mathrm{C}$. The fatty acid composition of this lipid fraction was deter mined by procedures we have described prevlously (19).

An aliquot of the lipid fraction was placed on a Silica gel HR plate, previously activated at $100^{\circ} \mathrm{C}$ for 30 minutes. The developing solvent was chloroform: methanol: acetic acid: 0.9 s saline $(75: 25: 10: 6)$. The separated lipids were visualized after exposure to $I_{2}$ vapors. The area migrating parallel to an authentic phosphatidyicholine was scraped from the TLC plate. The fatty acid composition of the various phospholipids was determined by gas
llquid chromatography of the methyl esters of the fatty acids (19). Each analysis was performed in duplicate.

PAPase activity was measured by a modification of the method of Coleman and Hübscher (9). Inorganic phosphate was quantified by the method described by Bartlett (1). The amniotic fluid pellet fraction was further characterized by sucrose gradient centrifugation segaration. In this study, PAPase was substrate assay procedure (20). Protein content was measured by the method of Lowry et al.. (11). Total phospholipids and the phosphatidylcholine fractions were quantified by the procedure described by Parker and Peterson (14).

\section{RESULTS}

The distribution of the activity of PAPase as well as that of the quantity of phospholipid, paimitate and protein are presented in Table 1. The results obtained from the analyses of the supernatant fraction, following centrifugation at $105,000 \times \mathrm{g}$ for 60 minutes, and for the pellet of this centrifugation are expressed as percentages of that found in the original sample. Amniotic fluids from six gravidas were analyzed and the tabulated results are the average of these six samples. From these data it is apparent that most of the PAPase activity was found primarily in the $105,000 \times \mathrm{g}$ pellet. In some samples, as much as 908 of the PAPase activity of the original sample was found in the pellet. Similarly, the phospholipid was also found principally in the pellet fraction. Of the total palmitate present in the original sampl (700 $\times \mathrm{g}$ supernatant fraction), approximately 908 was found in the pellet
after $105,000 \times \mathrm{g}$ centrifugation. While the PAPase, phospholipids, and palmi tate content was found in the pellet, by sharp contrast, only a small amount of the total protein was pelletized by 60 minutes of centrifugation at 105, 000 $\times \mathrm{g}$.

The phospholipid present in the $105,000 \times \mathrm{g}$ pellet fraction was further

The results of these studies are presented In Table 2. Of the phospholipld in the pellet, $78 \%$ was phosphatidylcholine. The phosphatidyicholine was further analyzed to ascertain the fatty acld distribution. Of the total fatty acids assoclated with the phosphatidylcholine $80 \%$ was palmitic acid.
The finding of thls disproportionately high concentration of phosphatidylcholine together with the marked enrichment of this fraction with palmitate strongly suggests that the material being sedimented from amnlotic fluid by high speed centrifugation was similar if not identical to lung surface-active material.

By a sucrose gradient centrifugation system, the resuspended pelletized material obtained from amniotic fluid layered as a band at a density simliar to that reported for both lamellar bodies (20) and surfactant (15). Further more, the activity of PAPase was predominately localized in this band and the specific activity of PAPase in this fraction was similar to that found in the material pelletized by high speed centrifugation of amniotic fluid was surface-active material.
Several lines of evidence gathered from this study give strong support to the concept that the release of the surface-active material by the fetal

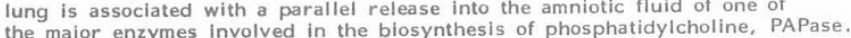
the major enzymes involved in the biosynthesis of phosphatidylcholine, PAP
Moreover, the PAPase activity and the surface-active material were found Moreover, the PAPase activity and the surface-active material were found in the same fractions. In other studies we have also shown that lamellar bodies isolated from lung tissue also contain a significant amount of PAPase (20) and CPTase (21). In these stud'? Is it was observed that the specific activity of PAPase in lamellar bodies was the highest of any of the various
subcellular organelles isolated from lung tissue $(20)$. The pelletized fraction subcellular organelles isolated from lung tissue $(20)$. The pelletized fraction isolated from the amniotic fluid after $105,000 \times \mathrm{g}$ centrifugation closely resemble
the lamellar bodies in (1) its phospholipid pattern, (2) palmitate concentration the lamellar bodies in (1) its phospholipid pattern, (2) palmitate concentration
in phosphatidylcholine; and, (3) the presence of PAPase activity of high in phosphatidylcholine; and, (3) the presence of PAPase activity of high specific activity. The density of the pellet obtained from amniotic fluid of term gestations was also found to be similar to that of lamellar bodies isolated
from lung tissue and similar also to that of the surface-active materials isolated by lung lavage. Each of these components, i.e. Isolated lamellar bodies, surface-active material Isolated by lung lavage and the $105,000 \times \mathrm{g}$ pellet
of human amniotic fluid were found to contain PAPase activity of high specific activity. Collectively, these findings provide strong support for the concept that a lamellar-like body is released from the fetal lung into the amniotic fluid. The results of previous studies by Novy, et al.. (13) of the pellets obtained following centrifugation of amniotic fluid from term gestations monkeys also indicate that a lamellar-like structure to be present in the amniotic fluid
of this species. The chemical and enzymatic studies of human amniotic fluid reported herein, together with the findings of Novy and colleagues, that include electron micrographs, constitute convincing evidence that the fraction obtained by centrifugation is of lung origin. This obtains since the physical and chemical data as well as the morphologic studies strongly resemble those characteristic of lamellar bodies isolated from lung tissue. The finding of such a close association of the activity of PAPase with both the surface-active material in the lamellar bodies and the surface-active material found in amniotic fluid is also consistent with the view that these materials enter the amniotic fluid as an associated particle. Previously we have reported studies in which
it was demonstrated that human amniotic fluid PAPase was of fetal lung origin (10). Novy, et al. (13) found that the lamellar bodles present in monkey amniotic fluid $\bar{w} \overline{r e}$ of fetal lung origin. Together these findings negate the
possibility that PAPase and lamellar bodies were released into amniotic fluid from tissue sites other than the lung.

These results together with studies reported previously from our laboratory and others provide the foundation for a unified hypothesis. This concept is illustrated graphically in Figure 1 , in which the Type II lung cell, in association with the Type I pneumocyte, are depicted. The characteristic lamellar bodies containing the surface-active materlal are shown in the Type surface-active material (20). The presence of phosphatidylglycerol and its surface-active material (20). The presence of phosphatidylglycerol and its Previously it was considered that the membrane surrounding the lamellar bodies in the Type II cell was the site of enriched amounts of PAPase (12). However, from the results of this study we suggest that PAPase may be an
integral part of the lamellar body, possibly one of the several proteins known to be assoclated with the surface-active material. This view is based on the observation that the lamellar bodies isolated from the human amniotic fluid contain a significant quantity of PAPase, yet from the electron microscopic studies of Novy, et al, (13) a distinct perilamellar membrane was not discern: ible.

The surface-actlve material synthesized in the lamellar body can migrate to the alveolar surface, is extruded Into the alveolar space and thence by body to the cell surface occurs via the microtubule system. Fallure of extrusion of surfactant was demonstrated after use of inhibitors of microtubule formation (2). The development of respiratory distress in an adult woman, followed
(2) by death after the ingestion of a large dose of colchicine to commit suicide suggests that a similar mechanism for the release of surface-active material suggests that a similar mechanism for the release of surface-active materla could provide the necessary coating of the alveolar surface to prevent collapse.

This unified concept envisions that the lamellar body of the Type II lung cell is an organelle of biosynthetic capability. During the course of human gestation there is a marked increase in the biosynthetic activity of key enzymes in phosphatidylcholine synthesis, e.g., PAPase. This "surge"
in activity precedes the augmented synthesis of lecithin characteristic of fetal lung maturation. PAPase, which is intimately associated with the lamellar body, replete with surface-active material reaches the alveolar surface by the microtubular system. The perilamellar membrane fuses with the plasma membrane of the Type II cell, a release of surface-active material to the alveolar membrane of the Type II cell, a release of surface-active material to the alveola space occurs and it subsequently reaches the amniotic fluid by water movem
by fetal respiration in utero. This would account for the higher specific activity of the enzyme PAPase found in the nasopharyngeal fluid than in amniotic fluid.

\section{SUMMARY}

The distribution of PAPase, phosphatidylcholine, and palmitate has been measured in human amniotic fluld following centrifugation at 105,000
$x \mathrm{~g}$ for 60 minutes. Of the total PAPase activity in the original sample ( 700 $\times \mathrm{g}$ for 60 minutes. Of the total PAPase activity in the original sample $(700$ $\times$ g supernatant fraction), $67 \%$ was found in the pellet. Similarly, the phosph-
lipid was also found principally in the pellet fraction. Of the total palmitate present in the original sample, approximately $90 \%$ was precipitated in the pellet. The finding of such a close association of the activity of PAPase with the surface-active material found in the amniotic fluid is consistent with the view that these materials are secreted into the amniotic fluid as a closely assoclated particle.

\section{REFERENCES AND NOTES}

1. Bartlett, G. R.: Phosphorous assay in column chromatography. J. Biol,

Chem., 234: 466 (1959)

2. Delahunty, T. J. and Johnston, J. M.: The effect of colchicine and vinblastine on the release

3. Farrell, P. M., and Avery, M. E.: Hyaline membrane disease. Am. Rev,

4. Folch, J., Lees; M., and Sloane Stanley, G. H.: A simple method for 
the isolation and purification of total lipids from animal tissues. J, Biol. Chem., 226: 497 (1957)

5. Gluck, L., Sribney, M., and Kulovich, V.: The biochemical development of surface activity in mammalian lung. II. The biosynthesis of phosphiolipids in the lung of Res., 1: 247 (1967)

6. Hallman, M., and Gluck, L.: Phosphatidyl glycerol in lung surfactant: I. Synthesis in rat lung microsomes, Biochem. Biophys. Res. Commun., 60: $1(1974)$

7. Hill, R. N., Spragg, R. G., Wedel, M. K., and Moser, K. M.: Adult respiratory distress syndrome associated with colchicine intoxication. Ann. Int. Med., 83: 523 (1975).

8. Hübscher, G.: Ǵlyceride metabolism. In S. J. Wakil: Lipid Metabolism (Academic Press, New York, 1970).

9. Jimenez, J. M., Schultz, F. M., MacDonald, P. C., and Johnston, J.M. Fetal lung maturation: II. Phosphatidic acid phosphohydrolase in human amniotic fluid, Gynecol, Invest., 5: 245 (1974).

10. Jimenez, J. M. Schultz, F. M.. and Johnston, J. M.: Fetal lung maturation: iII. Amniotic fluid phosphatidic acid phosphohydrolase (PAPase) and Its relation to the lecithin/sphingomyelin ratio. Obstet. Gynecol., 46: 588 (1975)

11. Lowry, H. O., Rosenbrough, N. J., Farr, A. L., and Randall, R. J. Protein measurements with the Folin phenol reagent. J. Biol. Chem. 193: 265 (1951)

12. Meban, C.: Localization of phosphatidic acıd phosphatase activity in granular pneumonocytes. J. Cell. Blo., 53:249 (1972)

13. Novy, M. J.. Portman, O. W.., and Bell, M.: Evidence for pulmonary and other sources of amnlotic fluid phospholipids in the rhesus monkey. In C. A Villee, D, B VIIlee and J, Zuckerman: Respiratory Distress Syndrome (Academic Press, New York, 1973)

14. Parker, F., and Peterson, N. F.: Quantitative analysis of phospholiplds and phospholipid fatty acids from sillica gel thin-layer chromatograms.

J. Lipid Res. 6: 455 (1965).
15. Pruitt, K. M., Cherng, M. J., and Spltzer, H. L.: Physical and chemical characterization of pig lung surfactant lipoprotein. Arch. Int. Med.

26. Rooney, S. A.., Page-Roberts, B. A., and Motoyama, E. K.: Role of lamellar inclusions in surfactant production: studies on phospholipid composition and biosynthesis in rat and rabbit lung subcellular fractions. J. Lipid Res., 16:418 (1975).

17. Schachi, J., and Agranoff, B. W.: Acetylcholine stimulates hydrolysis of $\mathrm{P}^{32}$-labeled phosphatidic acld in guinea pigs synaptosomes. Biochem. Biophys. Res. Commun., 50: 934 (1973)

18. Schultz, F. M., Jimenez, J. M., MacDonald, P. C., and Johnston, J. M.. Fetal lung maturation: I. Phosphatidic acid phosphohydrolase in rabbit ung. Gynecol, Invest., 5: 222 (1974)

19. Schwarz, B. E., Schultz, F. M., MacDonald, P. C., and Johnston, J. M. Initiation of human parturition III. Fetal membrane content of prosta-

20. Splandin $E_{2}$ and $F_{2 \alpha}$ precursor. Obstet. Cynecol., 46: 564 (1975). Phospholipid blosynthesis in lung lamellar bodies. Biochem. Biophys. Res. Commun., 66:17 (1975)

21. Spitzer, H. L., Wallis, P. A., and Johnston, J. M.: Phosphatidylcholine biosynthesis by isolated lamellar bodies from lung type II cells.

22. Spitzer, H. L.. Jimenez, J. M., and Johnston, J. M.: Persona

23. Supported in part by PHS Grant 1 PO $1 \mathrm{HD} 0867,-01$ and USPHS Grant AM-03108.

24. The authors thank Mary Bob Wylle and Robert H. Trubey for their technical assistance In this investigation and gratefully acknowledge Jaye Patton for her assistance in the preparation of the manuscript.

25. Requests for reprints should be addressed to: Juan M. Jimenez, M.D., The University of Texas Health Science Center at Dallas, Southwestern Medical School, Department of Obstetrics and Gynecology, 5323 Harry Hines Boulevard, Dallas, Texas 75235, USA.

26. Accepted for publication May 5, 1976

TABLe 1

THE DISTRIBUTION OF COMPONENTS IN HUMAN

AMNIOTIC FLUID FOLLOWING CENTRIFUGATION

AT $105,000 \times \mathrm{g}$ FOR 1 HOUR

\begin{tabular}{lcccc}
\hline & PAPase & Phospholipid & Palmitate & Protein \\
\hline Supernatant & 33 & 18 & 11 & 96 \\
Pellet & 67 & 82 & 89 & 4
\end{tabular}

TABLE ॥

CHARACTERIZATION OF THE PHOSPHOLIPID AND FATTY ACID OF

PHOSPHATIDYLCHOLINE IN THE PELLET FRACTION OBTAINED

AFTER CENTRIFUGATION OF HUMAN AMNIOTIC FLUID

AT $105,000 \times$ g FOR 1 HOUR

Percent

Phospholipid

Phosphatidylcholine

Fatty acids present in

phosphatidylcholine

$\begin{array}{rr}14: 0 & 4 \\ 16: 0 & 75 \\ 18: 0 & 4 \\ 18: 1 & 11 \\ 18: 2 & 3 \\ 20: 4 & 3\end{array}$

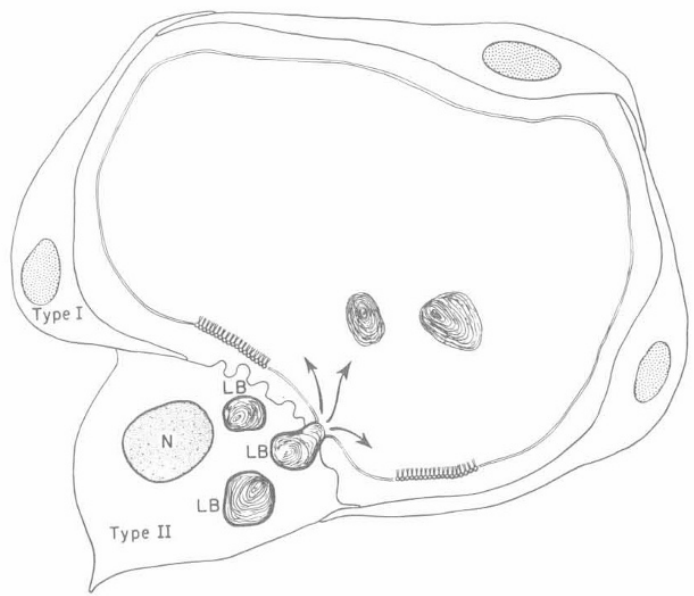

Figure 1. Schematic representation of an alveolus with type I and type II cells. The lamellar bodies formed in the type II cell, after being devoided of their perilamellar membrane are extruded into the alveolar space, where they may col the arvelar surface or are carried as a lamellar-like body into the amniotic fluid via the respiratory tract. 